\title{
The main galaxy groups from the SDSS data release $5^{\star}$
}

\author{
Xin-Fa Deng, Ji-Zhou He, Peng Jiang, Cheng-Hong Luo, and Ping Wu \\ School of Science, Nanchang University, Jiangxi 330031, PR China \\ e-mail: xinfadeng@163.com \\ Received 16 September 2006 / Accepted 3 August 2007 \\ ABSTRACT

\begin{abstract}
Aims. We investigate the correlations between galaxy properties and environment. $0.2 \times \bar{n}^{-1 / 3}=1.97 \mathrm{Mpc}$ by the three-dimensional friends-of-friends (FOF) algorithm, where $\bar{n}$ is the mean galaxy density, and compare basic properties of member galaxies of main galaxy groups with those of the main galaxy sample and isolated galaxies in each redshift bin.

Results. The luminosities and sizes of galaxies are almost independent of environment, but morphologies and colors are correlated with environment-especially galaxy morphologies.
\end{abstract} \\ Methods. From the main galaxy sample of the SDSS Data Release 5, we have extracted 11163 groups at the linking length $R_{0}=$
}

Key words. galaxies: fundamental parameters - cosmology: large-scale structure of Universe

\section{Introduction}

Galaxy groups have been a very important issue in discussions of the large-scale structure of the universe. In the hierarchical scenarios of structure formation of the universe, galaxy groups can be regarded as systems intermediate between individual galaxies, pairs and triplets and rich clusters. The information obtained from these systems allows us to understand many important issues better, for example, environmental studies.

The first sizeable sample of groups was constructed by Geller \& Huchra (1982), who identified 176 groups with three or more galaxies from the CfA galaxy redshift survey. In recent years, some large catalogs of groups have been compiled from different redshift surveys (Merchán et al. 2000; Giuricin et al. 2000; Tucker et al. 2000; Carlberg et al. 2001; Ramella et al. 2002; Merchán \& Zandivarez 2002; Eke et al. 2004; Gerke et al. 2005; Merchán \& Zandivarez 2005; Berlind et al. 2006), particularly from the Sloan Digital Sky Survey and 2 degree Field Galaxy Redshift Survey. Many authors also performed studies involving groups, ranging from local physical properties (Martínez et al. 2002; Domínguez et al. 2002; Ragone et al. 2004) to large scale structure (Zandivarez et al. 2003; Padilla et al. 2004).

In recent years, the Sloan Digital Sky Survey (SDSS) commissioning data is being widely used to explore properties of galaxy groups and clusters. Lee et al. (2004) identified compact groups in the SDSS Early Data Release (EDR; Stoughton et al. 2002) by using a modified version of Hickson's (1982) criteria. From the spectroscopic sample of the SDSS Second Data Release (DR2; Abazajian et al. 2004), Miller et al. (2005) extracted the "C4 Cluster Catalog". The $\mathrm{C} 4$ cluster-finding algorithm identifies clusters as overdensities in a seven-dimensional position and color space. Berlind et al. (2006) used a

^ Full Table 2 is only available in electronic form at the CDS via anonymous ftp to

cdsarc.u-strasbg.fr $(130.79 .128 .5)$ or via

http://cdsweb.u-strasbg.fr/cgi-bin/qcat?]/A+A/474/783 redshift-space friends-of-friends algorithm to identify galaxy groups and clusters in volume-limited samples of the SDSS redshift survey. This work aims to construct a group catalog from the main galaxy sample (Strauss et al. 2002) of the Sloan Digital Sky Survey (SDSS) Data Release 5 (Adelman-McCarthy et al. 2007) and investigate the correlations between galaxy properties and environment. The group identification is performed using the three-dimensional friends-of-friends (FOF) algorithm developed by Davis et al. (1985). Our paper is organized as follows. In Sect. 2, we describe the data used. The group identification algorithm is discussed in Sect. 3. In Sect. 4, we study the correlations between galaxy properties and environment. Our main results and conclusions are summarized in Sect. 5.

\section{Data}

The SDSS observes galaxies in five photometric bands $(u, g, r, i, z)$ centered at $(3540,4770,6230,7630$, $9130 \AA$ A). York et al. (2000) provided technical summary of the SDSS. The imaging camera was described by Gunn et al. (1998), while the photometric system and the photometric calibration of the SDSS imaging data were described by Fukugita et al. (1996), Hogg et al. (2001) and Smith et al. (2002). Pier et al. (2003) described the methods and algorithms involved in the astrometric calibration of the survey, and presented a detailed analysis of the accuracy achieved. Many of the survey properties were discussed in detail in the Early Data Release paper (Stoughton et al. 2002). The main galaxy sample (Strauss et al. 2002) targets galaxies brighter than $r_{\mathrm{p}}<17.77$ ( $r$-band apparent Petrosian magnitude). Most galaxies of this sample are within the redshift region $0.02 \leq z \leq 0.2$.

The spectroscopic area of the SDSS DR5 (AdelmanMcCarthy et al. 2007) is 5740 sq deg, which can be separated into three regions. Two of them are located north of the Galactic plane, one of which is at the celestial equator and the other at high declination. The third lies south of the Galactic plane, a set 
of three stripes near the equator. Each of these regions covers a wide range of survey longitude.

The data was downloaded from the Catalog Archive Server of the SDSS Data Release 5 (Adelman-McCarthy et al. 2007) by the SDSS SQL Search (with SDSS flag: bestPrimtarget $=64$ ) with high-confidence redshifts (Zwarning $\neq 16$ and Zstatus $\neq 0,1$ and redshift confidence level: $z_{\text {conf }}>0.95$, http://www.sdss.org/dr5/). From this sample, we select 332412 main galaxies in the redshift region $0.02 \leq z \leq 0.2$.

In calculating the co-moving distance we use a cosmological model with a matter density $\Omega_{0}=0.3$, cosmological constant $\Omega_{A}=0.7$, Hubble's constant $H_{0}=100 h \mathrm{~km} \mathrm{~s}^{-1} \mathrm{Mpc}^{-1}$ with $h=0.7$.

\section{The group-finding algorithm}

For group identification, the friend-of-friend (FoF) algorithm developed by Huchra \& Geller (1982) is the most frequently applied method for redshift surveys. The FOF algorithm starts with any galaxy as the beginning of a trial group. A search for companions around the starting galaxy is carried out with the following criteria:

$D_{i j}=2 \sin \left(\frac{\theta_{i j}}{2}\right) D \leq D_{L}=D_{0} R_{L}$

and

$V_{i j}=\left|V_{i}-V_{j}\right| \leq V_{L}=V_{0} R_{L}$,

where $D_{i j}$ is the projected distance, $\theta_{i j}$ the angular separation, $D=\left(D_{i}+D_{j}\right) / 2$ the mean radial comoving distance, and $V_{i j}$ the line-of-sight velocity difference. All companions and the starting galaxy are added to the group. Then, each of the new group members is in turn used as a center to search for its companions to add to the growing group, continuing until no more sufficiently close new galaxies are found.

To compensate for the number density variation due to the apparent magnitude limit of the survey, the scaling factor $R_{L}$ is computed by using the galaxy luminosity function of the sample $\Phi(M)$ :

$R_{L}=\left[\frac{\int_{-\infty}^{M_{12}} \Phi(M) \mathrm{d} M}{\int_{-\infty}^{M_{\lim }} \Phi(M) \mathrm{d} M}\right]^{-1 / 3}$

where

$M_{\mathrm{lim}}=m_{\mathrm{lim}}-25-5 \log D_{\mathrm{f}}$,

$M_{12}=m_{\lim }-25-5 \log D$,

$D_{\mathrm{f}}$ and $D$ are the fiducial and mean galaxy distances, respectively. $D_{0}$ and $V_{0}$ are the projected distance and the line-of-sight velocity difference at a fixed fiducial distance, respectively.

Many authors modified the Huchra-Geller's FoF algorithm, for example, Berlind et al. (2006) used constant linking lengths:

$D_{\perp, i j}=\left(c / H_{0}\right)\left(z_{i}+z_{j}\right) \sin \left(\frac{\theta_{i j}}{2}\right) \leq b_{\perp} \bar{n}^{-1 / 3}$,

$D_{\|, i j}=\left(c / H_{0}\right)\left|z_{i}-z_{j}\right| \leq b_{\|} \bar{n}^{-1 / 3}$,

where $\bar{n}$ is the mean number density of galaxies, and $b_{\perp}$ and $b_{\|}$ are the projected and line-of-sight linking lengths in units of the mean intergalaxy separation. Berlind et al. (2006) selected linking lengths that are best at grouping galaxies that occupy the same dark matter halos. These values are: $b_{\perp}=0.14, b_{\|}=0.75$, which are different to those used in previous FoF group analyses. Additionally, Berlind et al. (2006)' study also showed that there are no values for linking lengths that can pass all tests, and the right choice of linking lengths depends on the scientific objectives of the work.

The main goal of this paper is to explore the influence of spatial density enhancement on galaxy properties. With this objective in mind, we apply the simplest friends-of-friends algorithm to identify galaxy groups, which only use the spatial positions of galaxies in redshift space to identify groups, and not galaxy properties such as color or luminosity. By allowing a longer linking length in the radial direction, the Huchra-Geller FoF algorithm successfully accounted for redshift-space distortions. But $D_{0}$ and $V_{0}$ values adopted by most authors made the criterion of radial distance far larger than that of the projected separation. We do not know the proper ratio of the velocity dimension to the projected spatial dimension in order to correct redshift-space distortions. If the ratio of the criterion of radial distance to that of the projected separation is far larger than the proper ratio for correcting redshift-space distortions, groups identified by such a method are seriously contaminated by background/foreground galaxies. In this paper, we try two approaches: the Huchra \& Geller (1982) algorithm and the friends-of-friends (FOF) algorithm of Davis et al. (1985) which defines the three-dimensional linking length as $b \times n^{-1 / 3}$ where $\bar{n}$ is the mean particle density, in order to identify groups within $N$-body simulations. An attractive feature of Davis et al. (1985)'s method is that it does not impose any fixed shape on groups. For relatively large values of $b$, the resulting groups are often quite irregular with several separate centers of concentration, while smaller values of $b$ tend to identify groups with a well-defined center and relatively regular structure. Davis et al. (1985) used $b=0.2$ to identify dark matter groups. Jenkins et al. (2001)'s study showed that this choice of linking length yields a halo mass function that is independent of redshift and $\Omega_{0}$, and thus provides a good definition of the underlying dark matter haloes.

Though Davis et al. (1985)'s algorithm became three dimensional and thus less subject to projection effects, this algorithm does not take into account the stretching of groups in redshift space along the radial direction-redshift space distortions. But when we have no ability to correct redshift-space distortions, we must face the choice between two effects: the projection effects or redshift space distortions. Most authors selected the former, while it is the latter which was considered by Davis et al. (1985)'s algorithm. Undoubtedly, it is of interest to explore the properties of groups by using independent and different methods.

Using $b=0.2$ which corresponds to the linking length $R_{0}=1.97 \mathrm{Mpc}$ (for the main galaxy sample, the mean galaxy density is about $1.05 \times 10^{-3} \mathrm{Mpc}^{-3}$ ), we extract 11163 groups with richness $N \geq 4$, in which the richest group contains 113 galaxies. The whole group sample contains 73322 galaxies. Figure 1 shows the number of groups with different richness. We divide the range of richness of groups into 6 subintervals: $4 \leq N \leq 10 ; 10<N \leq 20 ; 20<N \leq 30 ; 30<N \leq 40 ; 40<$ $N \leq 50 ; N>50$, where $N$ is the member galaxy number in each group. As seen in Fig. 1, 89.35\% of groups are in Bin 1, and only about $0.10 \%$ in Bin 6 . Most of the groups are small and dense systems, typically containing four members. Figure 2 also shows the redshift distributions of the main galaxy sample (solid line) and member galaxies of groups (dashed line). In the low redshift 


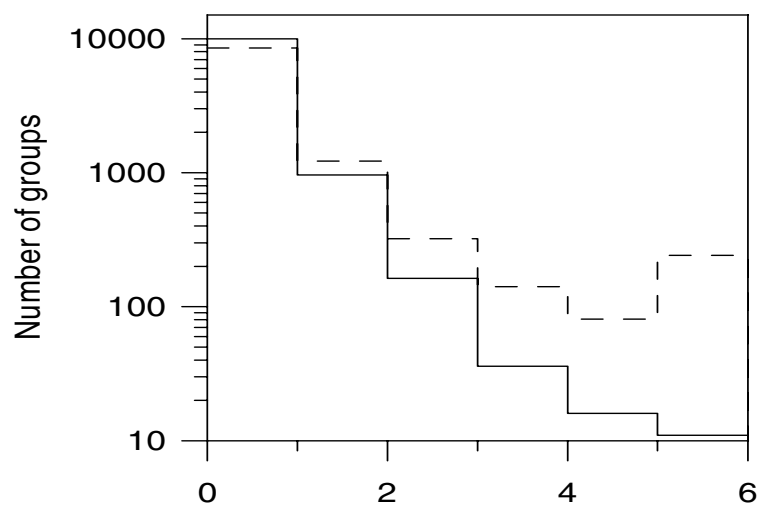

Fig. 1. The number of groups with different richness for the group cata$\log$ identified using the linking length $b=0.2$ (solid line) and the group catalog identified using the linking lengths $b_{\perp}=0.14, b_{\|}=0.75$ (dashed line).

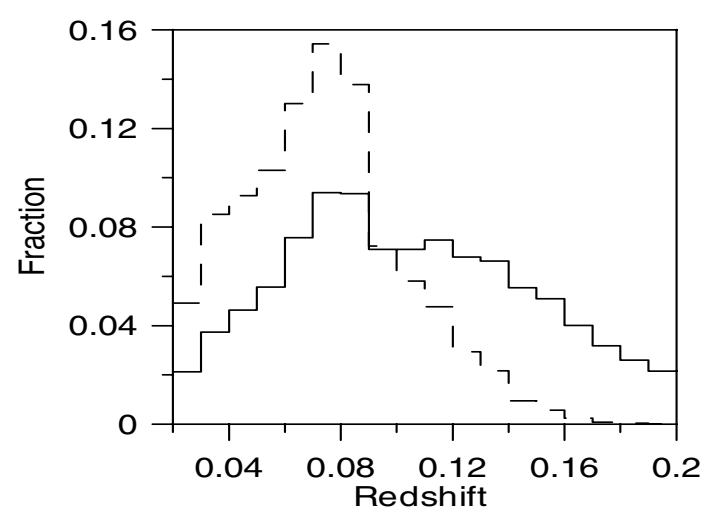

Fig. 2. The redshift distributions of the main galaxy sample (solid line) and member galaxies of groups identified using the linking length $b=$ 0.2 (dashed line).

region $(0.02 \leq z \leq 0.10)$, the group sample has a higher proportion of galaxies. The peak of the distribution is at about $z=0.08$. Because the main galaxy sample is an apparent-magnitude limited sample, the density of galaxies drops off with distance. This kind of the incompleteness of the parent sample will exert certain influence upon the result of identifying groups. In the dense region (low redshift region $0.02 \leq z \leq 0.10$ ) more groups are identified. In addition, we also notice that a compact super-largescale structure - the Sloan Great Wall (Gott et al. 2005; Deng et al. 2006a) is located around the peak value $z=0.08$.

As indicated by Berlind et al. (2006), there are two main sources of incompleteness in SDSS group catalogs: fiber collisions and survey edges, which will prevent galaxies from being included in some groups, cause the richness of these groups to be underestimated, and affect the multiplicity function of groups. But when we use a group sample to explore the dependence of galaxy properties on environment, the influence of such imcompleteness is not crucial. In addition, we also notice that correcting of some incompleteness often results in new bias. For example, Berlind et al. (2006) corrected for fiber collisions by giving each collided galaxy the redshift of its nearest neighbor on the sky (usually the galaxy it collided with). Putting collided galaxies at the redshifts of their nearest neighbors will cause some nearby galaxies to be placed at high redshift, which artificially makes their estimated luminosities very high. In addition, along with the availability of larger catalogs of galaxies, the influence of survey edges will become less important. Therefore, in this paper, we do not make efforts to correct incompleteness in SDSS group catalogs.

We also use the Huchra \& Geller (1982) algorithm and linking lengths adopted by Berlind et al. (2006): $b_{\perp}=0.14, b_{\|}=0.75$, to see if it changes the results. Thus, two criteria of group identification are:

$D_{\perp, i j}=2 \times \sin \left(\frac{\theta_{i j}}{2}\right) \times D \leq 0.14 \times \bar{n}^{-1 / 3}$,

$D_{\|, i j}=\left|D_{i}-D_{j}\right| \leq 0.75 \times \bar{n}^{-1 / 3}$,

where $D=\left(D_{i}+D_{j}\right) / 2$ is the mean radial comoving distance, and $\left|D_{i}-D_{j}\right|$ the radial comoving distance difference. We extract a total of 10522 groups with richness $N \geq 4$. There are 166809 member galaxies in groups, about $50.2 \%$ of the total galaxy number in the main galaxy sample. This proportion is apparently larger than that identified using the linking length $b=0.2$, and also larger than that identified by Berlind et al. (2006), using the same linking lengths. In Berlind et al. (2006), $37.2 \%, 40.6 \%$, and $42.3 \%$ of galaxies are in groups of three or more members for three samples.

At the linking lengths: $b_{\perp}=0.14, b_{\|}=0.75$, in the region at the celestial equator of the North of the Galactic plane, a huge system is formed, which contains 41806 galaxies, about $45.4 \%$ of the galaxy number in this region (the total galaxy number in this region is 92058). This shows that filamentary morphology is the main structure of the distribution of galaxies in this region, in agreement with previous studies (Gott et al. 2005; Deng et al. 2006a). Gott et al. (2005) found that in this region, there is a huge Great Wall of galaxies, with a length of about $450 \mathrm{Mpc}$ in comoving coordinates. But in the region at high declination north of the Galactic plane, the richest group only contains 931 galaxies, about $0.46 \%$ of the galaxy number in this region (the total galaxy number in this region is 200374). These results show that for group identification the choice of linking lengths may depend on structure properties of the spatial distribution of galaxies. For the galaxy sample with filamentary morphology, the above linking lengths are too large, and many groups will be fused together into a huge system. The richest group identified using the linking length $b=0.2$ only contains 113 galaxies, which is far smaller than that identified using the linking lengths $b_{\perp}=0.14, b_{\|}=0.75$. As seen from Fig. 1, group catalogs identified using the linking length $b=0.2$ and the linking lengths $b_{\perp}=0.14, b_{\|}=0.75$ have different richness distribution. In group catalog identified using the linking lengths $b_{\perp}=0.14, b_{\|}=0.75$, there is a higher proportion of loose groups and even of clusters.

Praton et al. (1997) argued that distortions in redshift space may enhance structures perpendicular to the line of sight, such as the Great Wall of galaxies, and that in the flux-limited galaxy samples such an effect becomes more apparent. In order to decrease selection effects, a simple method is to use a volume limited galaxy sample. The major problem of flux-limited galaxy samples is the magnitude selection effect: at large distances faint galaxies are not visible and faint distant groups cannot be detected. But in volume limited samples fainter galaxies are excluded at all distances from the observer. The price for this replacement is that a large fraction of the data is not used. In this study, we still use the apparent-magnitude limited main galaxy sample.

In order to compile the group catalog, we also estimate basic physical properties of groups such as velocity dispersion, virial radius, virial mass and crossing time. Apparently, these measures are heavily influenced by the relative values of the linking 
Table 1. The mean physical properties of the two group catalogs.

\begin{tabular}{ccccccc}
\hline \hline Sample & $N_{\text {grou }}$ & $N_{\text {gala }}$ & $\begin{array}{c}\bar{\sigma}_{v} \\
\left(\mathrm{~km} \mathrm{~s}^{-1}\right)\end{array}$ & $\begin{array}{c}\bar{R}_{V} \\
(\mathrm{Mpc})\end{array}$ & $\begin{array}{c}\bar{M}_{V} \\
\left(M_{\odot}\right)\end{array}$ & $H_{0} \bar{t}_{\mathrm{cr}}$ \\
\hline $\begin{array}{c}\text { identified at the linking length } b=0.2 \\
\text { identified at the linking lengths } b_{\perp}=0.14, b_{\|}=0.75\end{array}$ & 11163 & 73322 & 71.37 & 3.51 & $1.38 \times 10^{13}$ & 1.07 \\
\hline
\end{tabular}

parameters. The mean physical properties of two group catalogs are listed in Table 1.

The line-of-sight velocity dispersion $\sigma_{v}$ is estimated by

$\sigma_{v}=\frac{1}{1+\bar{z}} \sqrt{\frac{1}{N-1} \sum_{i=1}^{N}\left(c z_{i}-c \bar{z}\right)^{2}}$,

where $N$ is the number of galaxy members and $\bar{z}$ the mean redshift of the group. Figure 3 shows the velocity dispersion distribution of group catalogs identified using the linking length $b=0.2$ and the linking lengths $b_{\perp}=0.14, b_{\|}=0.75$, respectively. Using the linking length $b=0.2$ (corresponding to the three-dimensional linking length $R_{0} \sim 1.97 \mathrm{Mpc}$ ), we only select groups in which the redshift difference between member galaxies is less than 0.001 , corresponding to the minimum comoving radial distance difference $\Delta D(z) \sim 3.9 \mathrm{Mpc}$. Shorter linking length in the radial direction will result in low velocity dispersions of groups. The mean velocity dispersion $\left(\bar{\sigma}_{v}=\right.$ $71.36 \mathrm{~km} \mathrm{~s}^{-1}$ ) of the group catalog identified using the linking length $b=0.2$ is much smaller than that of other group catalogs(see Table 1 of Merchán \& Zandivarez 2005), also far smaller than that of the group catalog identified using the linking lengths $b_{\perp}=0.14, b_{\|}=0.75\left(\bar{\sigma}_{v}=211.88 \mathrm{~km} \mathrm{~s}^{-1}\right)$. Due to their high densities and low velocity dispersions, such groups may represent an environment where interactions and galaxy mergers are expected to be more prevalent than in loose groups and clusters. At the linking lengths $b_{\perp}=0.14, b_{\|}=0.75$, systems at different redshift will merge into loose groups and clusters and even superclusters, which have larger velocity dispersions.

The virial radius is estimated using the following equation

$R_{V}=\frac{\pi}{2} \frac{N(N-1)}{\sum_{i>j} R_{i j}^{-1}}$,

where $R_{i j}$ is the galaxy projected distances. Figure 4 shows the virial radius distribution of group catalogs identified using the linking length $b=0.2$ and the linking lengths $b_{\perp}=0.14$, $b_{\|}=0.75$, respectively. According to Merchán \& Zandivarez (2005), the mean virial radius of some previous group catalogs is $\sim 1.1 \mathrm{Mpc}$, much smaller than that of the group catalog identified using the linking length $b=0.2\left(\bar{R}_{V}=3.51 \mathrm{Mpc}\right)$. This is due to the linking length $b=0.2$ (corresponding to the threedimensional linking length $R_{0} \sim 1.97 \mathrm{Mpc}$ ) apparently being larger than the criterion of the projected separation used in other group catalogs. At the linking lengths $b_{\perp}=0.14, b_{\|}=0.75$, loose groups and clusters and even superclusters are formed. In addition, in a flux-limited galaxy sample, structures perpendicular to the line of sight are enlarged. These factors result in that groups identified using the linking lengths $b_{\perp}=0.14$ and $b_{\|}=0.75$ have large virial radius $\left(\bar{R}_{V}=4.91 \mathrm{Mpc}\right)$.

The virial mass is computed as

$M_{V}=\frac{3 \sigma_{v}^{2} R_{V}}{G}$

where $G$ is the gravitational constant. The mean virial mass of groups identified using the linking length $b=0.2$

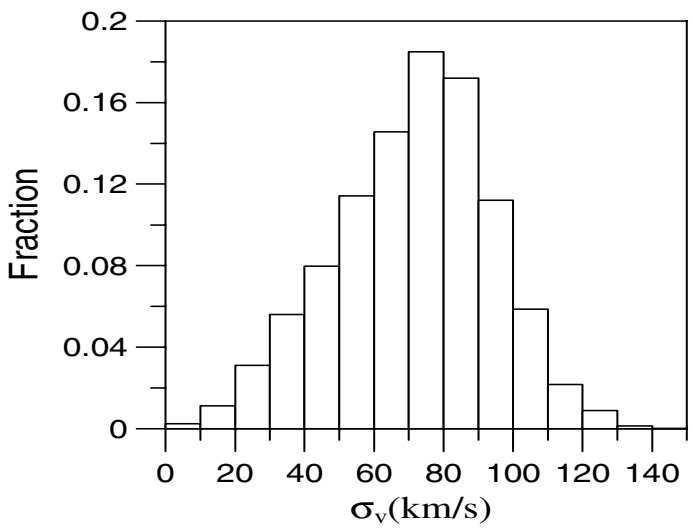

(a)

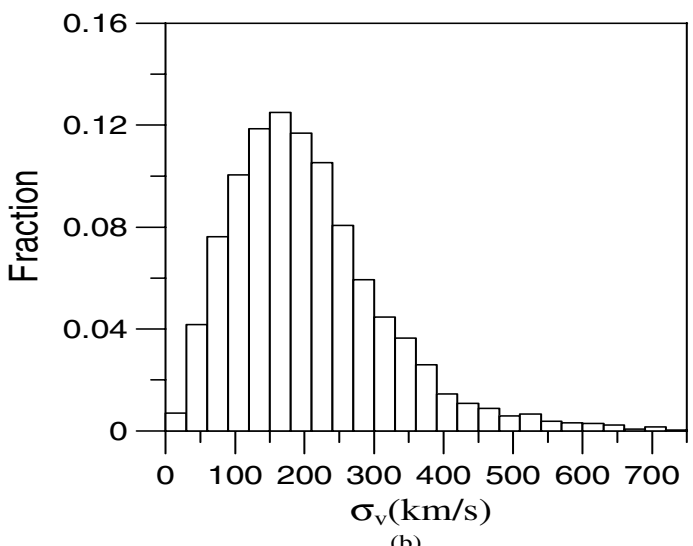

Fig. 3. Histogram of the velocity dispersion distribution of groups (a) identified using the linking length $b=0.2$, (b) identified using the linking lengths $b_{\perp}=0.14, b_{\|}=0.75$.

is $\bar{M}_{V}=1.38 \times 10^{13} M_{\odot}$, far smaller than that of other group catalogs (see Table 1 of Merchán \& Zandivarez 2005). Due to the formation of loose groups and clusters and even superclusters, the mean virial mass of groups identified using the linking lengths $b_{\perp}=0.14$ and $b_{\|}=0.75$ becomes quite large $\left(\bar{M}_{V}=2.52 \times 10^{15} M_{\odot}\right)$. Figure 5 illustrates the virial mass distribution of groups identified using the linking length $b=0.2$ and the linking lengths $b_{\perp}=0.14, b_{\|}=0.75$, respectively.

In order to test whether groups are virialized, we compute crossing times of groups and check whether they are sufficiently less than the Hubble time. The crossing time in units of the Hubble time $\left(H_{0}^{-1}\right)$ is defined as

$t_{\mathrm{cr}}=\frac{3}{5^{3 / 2}} \frac{R_{V}}{\sigma_{v}}$

Groups are systems of galaxies with crossing times $t_{\mathrm{cr}}$ much smaller than the Hubble time, indicating that collapse and virialization have been completed recently (Turner \& Sargent 1974; Jackson 1975; Gott \& Turner 1977; Rood \& Dickel 1978; Diaferio et al. 1993; Girardi \& Giuricin 2000; Tucker et al. 2000; Giuricin et al. 2001; Berlind et al. 2006). The short crossing 


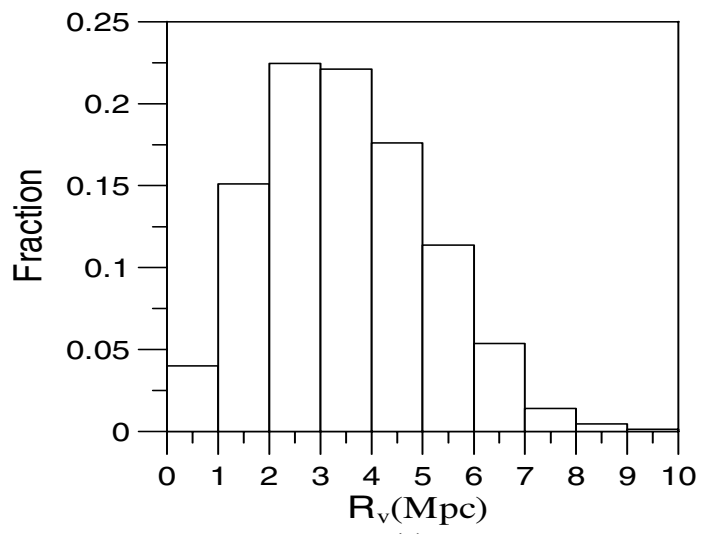

(a)

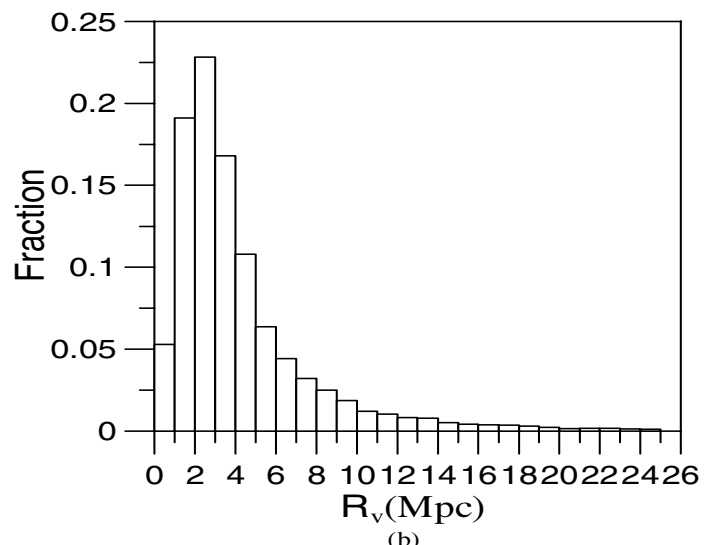

Fig. 4. Histogram of the virial radius distribution of groups (a) identified using the linking length $b=0.2$, (b) identified using the linking lengths $b_{\perp}=0.14, b_{\|}=0.75$.

time has been taken as an indication that the groups are in virial equilibrium. Figure 6 illustrates the crossing time distribution of groups identified using the linking length $b=0.2$ and the linking lengths $b_{\perp}=0.14, b_{\|}=0.75$, respectively. About $17.9 \%$ of groups identified using the linking length $b=0.2$ have crossing times less than $0.5 H_{0}^{-1}$, which is much smaller than the fraction of groups identified using the linking lengths $b_{\perp}=0.14, b_{\|}=$ $0.75(68.0 \%)$. One may wonder whether these groups are virialized systems or not. In Berlind et al. (2006) (using the same criteria), $80 \%$ of all groups have crossing times less than $\sim 0.29 \mathrm{H}_{0}^{-1}$, which suggested that most of their groups are likely virialized systems.

The crossing time is actually influenced by the relative values of the linking parameters, $D_{0}$ (which determines $R_{V}$ ) and $V_{0}$ (which determines $\sigma_{v}$ ). The short crossing times are biased towards cylindrical groups which spread out along the line of sight. This seems to correct redshift-space distortions, but according to the above analysis, it also results in projection effects in three dimensional space. In addition, Diaferio et al. (1993) indicated that the interpretation of the short crossing time may be incorrect.

According to the above analyses, Davis et al. (1985)'s algorithm is more reasonable for identifying groups in a flux-limited galaxy sample. We compute the unweighted centroid of each group, which consists of a group right ascension, declination, and mean redshift. Table 2 lists the catalog of groups identified using the linking length $b=0.2$. For each group, we list a group No. (Col. 1); the group richness $N$ (Col. 2); the right ascension and declination of the group centroid (Cols. 3, 4); the mean redshift (Col. 5); the line-of-sight velocity dispersion $\sigma_{v}$
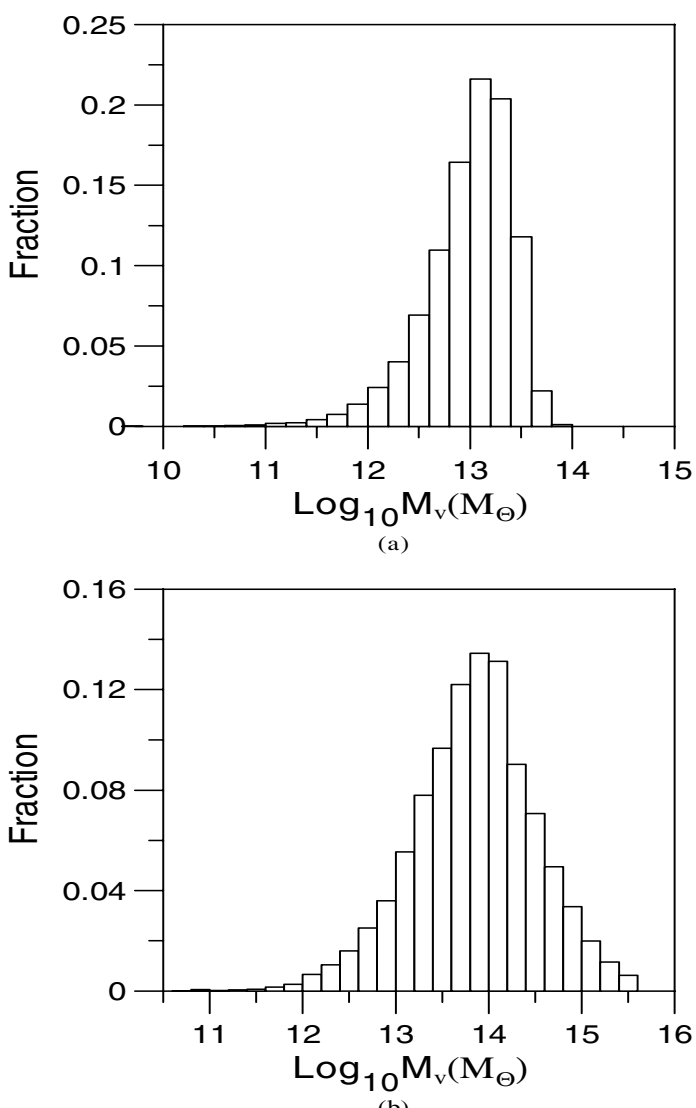

(b)

Fig. 5. Histogram of the virial mass distribution of groups (a) identified using the linking length $b=0.2$, (b) identified using the linking lengths $b_{\perp}=0.14, b_{\|}=0.75$.

(Col. 6); the virial radius $R_{V}(\mathrm{Col} .7)$; the virial mass $M_{V}(\mathrm{Col} .8)$; the crossing time $H_{0} t_{\mathrm{cr}}$ (Col. 9). We show the first few rows of Table 2 in the text and make the entire table available in the electronic version of the journal (available at the CDS).

\section{Correlations between galaxy properties and environment}

The Hickson (1982) analyses showed that the proportion of spiral galaxies decreases from $60 \%$ in the least compact groups to $20 \%$ in the most compact. In order to explore properties of galaxies in groups depending on the compactness of groups, we also identify two other group catalogs at the linking lengths $b=$ 0.15 and $b=0.25$. At the linking length $b=0.15,8347$ galaxy groups (with richness $N \geq 4$ ) containing 50006 galaxies are extracted, in which the richest group contains 98 galaxies, while at the linking length $b=0.25$, we identify 13395 galaxy groups (with richness $N \geq 4$ ) containing 95706 galaxies, in which the richest group contains 196 galaxies.

Luminosity, size, color and morphological type are the most basic properties of a galaxy. In this paper, the absolute magnitude $M_{r}$ is calculated from the $r$-band apparent Petrosian magnitude by using a polynomial fit formula (Park et al. 2005) of the K-correction (Blanton et al. 2003a) within $0<z<0.3$ :

$K(z)=2.3537(z-0.1)^{2}+1.04423(z-0.1)-2.5 \log (1+0.1)$.

$R_{50}$ and $R_{90}$ are the radii enclosing $50 \%$ and $90 \%$ of the Petrosian flux, respectively. We use the $r$-band $R_{50}\left(R_{50, r}\right)$ as the parameter of galaxy size. The concentration index $c_{i}=R_{90} / R_{50}$ is used to 
Table 2. The group catalog identified using the linking length $b=0.2$.

\begin{tabular}{ccccccccc}
\hline \hline $\begin{array}{c}\text { Group } \\
\text { No. }\end{array}$ & $N$ & $\begin{array}{c}\text { RA } \\
(\mathrm{deg})\end{array}$ & $\begin{array}{c}\text { Dec } \\
(\mathrm{deg})\end{array}$ & $\bar{z}$ & $\begin{array}{c}\sigma_{v} \\
\left(\mathrm{~km} \mathrm{~s}^{-1}\right)\end{array}$ & $\begin{array}{c}R_{V} \\
(\mathrm{Mpc})\end{array}$ & $\begin{array}{c}M_{V} \\
\left(M_{\odot}\right)\end{array}$ & $H_{0} t_{\text {cr }}$ \\
\hline 1 & 6 & 146.68663 & -0.73077 & 0.06492 & 53.81 & 2.63 & $5.31 \times 10^{12}$ & 0.92 \\
2 & 38 & 151.07102 & 1.04821 & 0.04600 & 86.15 & 8.01 & $4.15 \times 10^{13}$ & 1.75 \\
3 & 9 & 150.33435 & -0.07045 & 0.09201 & 68.71 & 5.13 & $1.69 \times 10^{13}$ & 1.40 \\
4 & 5 & 150.21042 & -0.22839 & 0.08796 & 74.77 & 4.11 & $1.60 \times 10^{13}$ & 1.03 \\
5 & 24 & 151.05738 & 0.52548 & 0.04500 & 82.75 & 5.83 & $2.78 \times 10^{13}$ & 1.32 \\
\hline
\end{tabular}

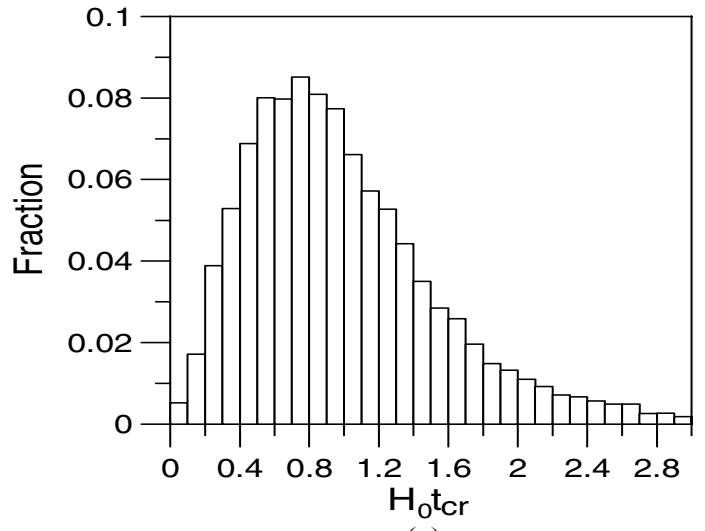

(a)

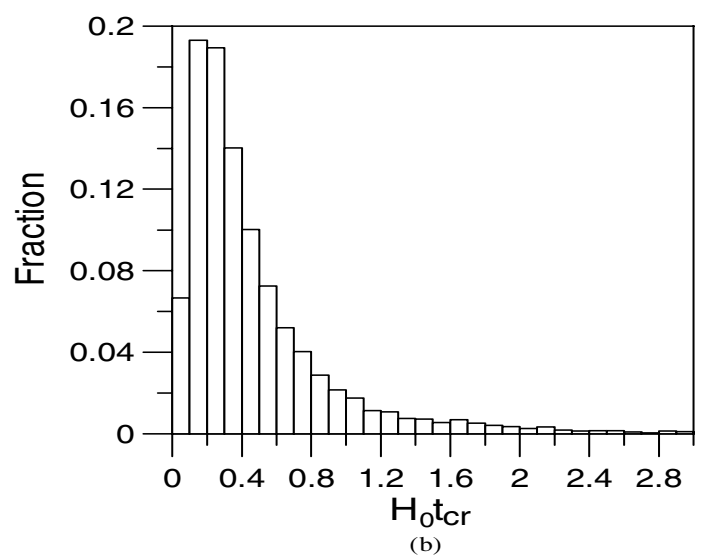

Fig. 6. Histogram of the crossing time distribution of groups (a) identified using the linking length $b=0.2$, (b) identified using the linking lengths $b_{\perp}=0.14, b_{\|}=0.75$.

separate early-type (E/S0) galaxies from late-type (Sa/b/c, Irr) galaxies (Shimasaku et al. 2001). As is well-known, the galaxy morphology is closely correlated with many other parameters, such as color and concentration index. These parameters can be used as the morphology classification tool (e.g., Park \& Choi 2005; Yamauchi \& Goto 2005; Abraham et al. 2003; Strateva et al. 2001; Shimasaku et al. 2001). The concentration parameter is a simple morphological parameter. Nakamura et al. (2003) showed that $c_{i}=2.86$ separates galaxies at S0/a with a completeness of about 0.82 for both late and early types.

Many properties of galaxies depend strongly on luminosity (e.g. de Vaucouleurs 1961; Kormendy 1977; Bower et al. 1992; Blanton et al. 2003b; Shen et al. 2003; Baldry et al. 2004; Balogh et al. 2004a; Kelm et al. 2005), for example, more luminous galaxies are redder. The main galaxy sample is an apparentmagnitude limited sample, in which the number of bright galaxies increases with increasing redshift $z$. In such a sample, the mean luminosity and many other properties of the galaxies apparently change with redshift $z$. So, we divide the whole redshift region into 18 bins of width 0.01 , and perform comparative studies of galaxy statistical properties among different samples in each redshift bin.

Figure 7 shows galaxy statistical properties as a function of redshift $z$ for member galaxies of groups identified at the linking lengths $b=0.2, b=0.25$ and $b=0.15$. Error bars represent standard deviation in each redshift bin for member galaxies of groups identified at the linking length $b=0.2$. We do not observe as large a statistical difference as Hickson (1982) for systems having different compactness. Three group samples have almost the same statistical properties. In the high redshift region $0.18 \leq z \leq 0.2$, the member galaxies of groups identified at the linking length $b=0.15$ apparently have different early-type proportions. Because the number of groups identified in the high redshift region is small, analysing results in this redshift region may lack high significance.

Isolated galaxies are special and rare galaxies in the universe. They often reside in the most underdense regions, and an interesting sample for studies of the effects of underdense environment on galaxy properties (Adams et al. 1980; Haynes \& Giovanelli 1980; Haynes et al. 1984; Koopmann \& Kenney 1998; Rojas et al. 2004; Hoyle et al. 2005; Rojas et al. 2005). In order to explore correlations between galaxy properties and environment, we perform comparative studies of galaxy statistical properties among groups, isolated galaxies and the main galaxy sample.

According to Karachentseva (1973)'s selection algorithm, a galaxy $i$ with angular diameter $a_{i}$ is considered isolated if the projected sky separation $x_{i, j}$ between this galaxy and any neighboring galaxy $j$ of angular diameter $a_{j}$ satisfies the following two criteria: $x_{i, j} \geq 20 \times a_{j} ; \frac{1}{4} \times a_{j} \leq a_{i} \leq 4 \times a_{j}$. Allam et al. (2005) modified the Karachentseva criteria, and used the following criteria: $x_{i, j} \geq 40 \times R_{j} ;\left|g_{i}-g_{j}\right|>3.0$, where $g_{i}, g_{j}$ are the $g$-band magnitude of galaxy $\mathrm{i}$ and galaxy $j$, and $R_{j}$ is the Petrosian radius of any neighboring galaxy $j$. These methods are based mainly on the two-dimensional projected sky separation and galaxy diameter. The isolated galaxy catalog identified by these methods is a two-dimensional sample. In this paper, we use three-dimensional cluster analysis (Einasto et al. 1984) and extract isolated main galaxies from the main galaxy sample. By cluster analysis, the sample can be separated into isolated galaxies, close double and multiple galaxies, galaxy groups or clusters. At larger radii, most galaxies of the sample form groups or clusters, and few galaxies are isolated. These isolated galaxies should be a good sample for studies of three-dimensional isolated galaxies. According to Deng et al. (2006b), isolated galaxies identified at dimensionless radius $r \geq 1.2$ (dimensionless radii $r=R / R_{1}, R_{1}=(3 /(4 \pi \times \bar{n}))^{1 / 3}$ is the radius of the sphere with unit population) can be defined as genuinely isolated in three-dimensional space. In this paper, we extract 14966 isolated galaxies at dimensionless radius $r=1.4\left(R_{1}\right.$ for the main galaxy sample is $6.11 \mathrm{Mpc}$ ) from the main galaxy sample. We 

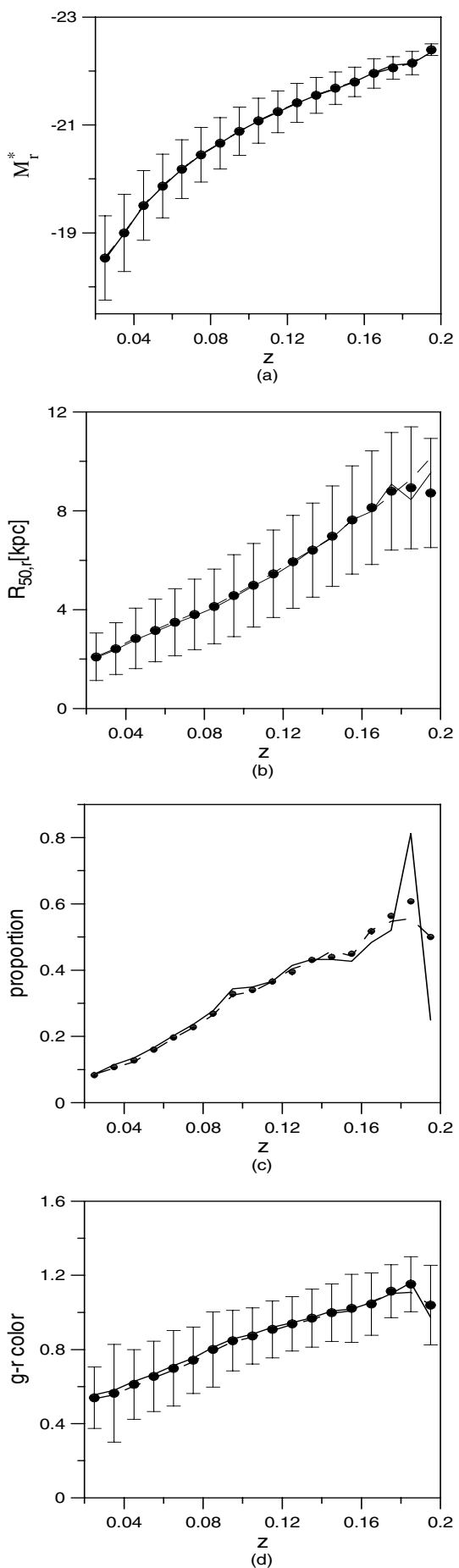

Fig. 7. Galaxy statistical properties as a function of redshift $z$ for member galaxies of groups identified at the linking lengths $b=0.2$ (dot), $b=0.25$ (dashed line) and $b=0.15$ (solid line) respectively. Error bars represent standard deviation in each redshift bin for member galaxies of groups identified at the linking length $b=0.2$ (a) mean luminosity, (b) mean size, (c) early-type proportion, (d) mean $g-r$ color.

note that at this radius systems having different sizes merge into a huge cluster network occuping most of the space of the sample and containing most galaxies. For example, in the region at high declination of the North of Galactic plane, the richest system forming at radius $r=1.4$ contains 147979 galaxies (about $73.85 \%$ of the total galaxy number in this region). Additionally, $R=1.4 \times R_{1}=8.55 \mathrm{Mpc}$ is larger than the three-dimensional distance criterion used by Rojas et al. (2004) to identify void galaxies which have fewer than three neighbors within $7 h^{-1} \mathrm{Mpc}$. It also implies a density contrast $\delta \rho / \rho<-0.64$ around isolated galaxies, which is lower than the density contrast $\delta \rho / \rho<-0.6$ around void galaxies. Therefore, isolated galaxies identified at $r=1.4$ are located in particularly low-density environments.

Galaxy properties are strongly correlated with environment, for example, galaxies in dense environments (i.e. clusters or groups) have a high proportion of early type morphologies (e.g. Oemler 1974; Dressler 1980; Whitmore et al. 1993; Deng et al. 2007) and low SFRs (e.g. Balogh et al. 1997, 1999; Poggianti et al. 1999). Many authors have explored correlations between environment and galaxy properties, such as the ones between environment and morphology (e.g. Postman \& Geller 1984; Dressler et al. 1997; Hashimoto \& Oemler 1999; Fasano et al. 2000; Tran et al. 2001; Goto et al. 2003; Helsdon \& Ponman 2003; Treu et al. 2003), between environment and star formation rate (e.g. Hashimoto et al. 1998; Lewis et al. 2002; Gómez et al. 2003; Balogh et al. 2004b; Tanaka et al. 2004; Kelm et al. 2005), and between environment and color (e.g. Tanaka et al. 2004; Balogh et al. 2004a; Hogg et al. 2004).

Clustering properties of galaxies strongly depend on galaxy luminosity: the most luminous galaxies exist preferentially in the densest regions of the universe (Davis et al. 1988; Hamilton 1988; Park et al. 1994; Loveday et al. 1995; Guzzo et al. 1997; Benoist et al. 1998; Norberg et al. 2001; Norberg et al. 2002; Zehavi et al. 2002; Blanton et al. 2003b). Applying the projected correlation functions $\omega_{p}\left(r_{p}\right)$, Zehavi et al. (2002) found that more luminous galaxies cluster more strongerly. Blanton et al. (2003b) showed that local density is a strong function of luminosity. By calculating the projected correlation functions of galaxies having different spectral types, Norberg et al. (2002) found that luminosity, and not type, is the dominant factor of galaxy clustering.

If structure in the Universe grows due to successive mergers of dark matter halos, and massive galaxies are the result of merging of smaller ones, then a high density of galaxies seems helpful to produce a bright galaxy. In Fig. 8a, we present the mean luminosity ( $r$-band) as a function of redshift $z$ for member galaxies of groups identified at the linking length $b=0.2$, the main galaxy sample (dashed line) and isolated galaxies (solid line). We do not observe a significant mean luminosity difference. This shows that the luminosity is not strongly correlated with environment. A similar conclusion was obtained by Deng et al. (2007). They compared statistical properties of galaxy luminosity in a compact galaxy group sample with those in a random group sample, and found that the two samples had the same statistical properties of luminosity. These results are not consistent with the previous conclusions.

In order to study the dependence of clustering on luminosity, Zehavi et al. (2002) used three volume-limited subsamples, each with different absolute magnitude and redshift limits. The space density of the three subsamples is different: the space density of the lowest luminosity subsample is 27 times that of the highest luminosity subsample. Their redshift ranges are also disjointed. In Zehavi et al. (2002)'s analysis, it is not easy to determine whether the difference of clustering of the three subsamples is due to their luminosity difference or other factors. Using the projected correlation function, there is a serious projection effect in such analyses, due to the lack of radial distance information. In fact, Zehavi et al. (2002)'s results demonstrated that the correlation length of the highest luminosity subsample is much larger than that of the lowest luminosity subsample, but did not 

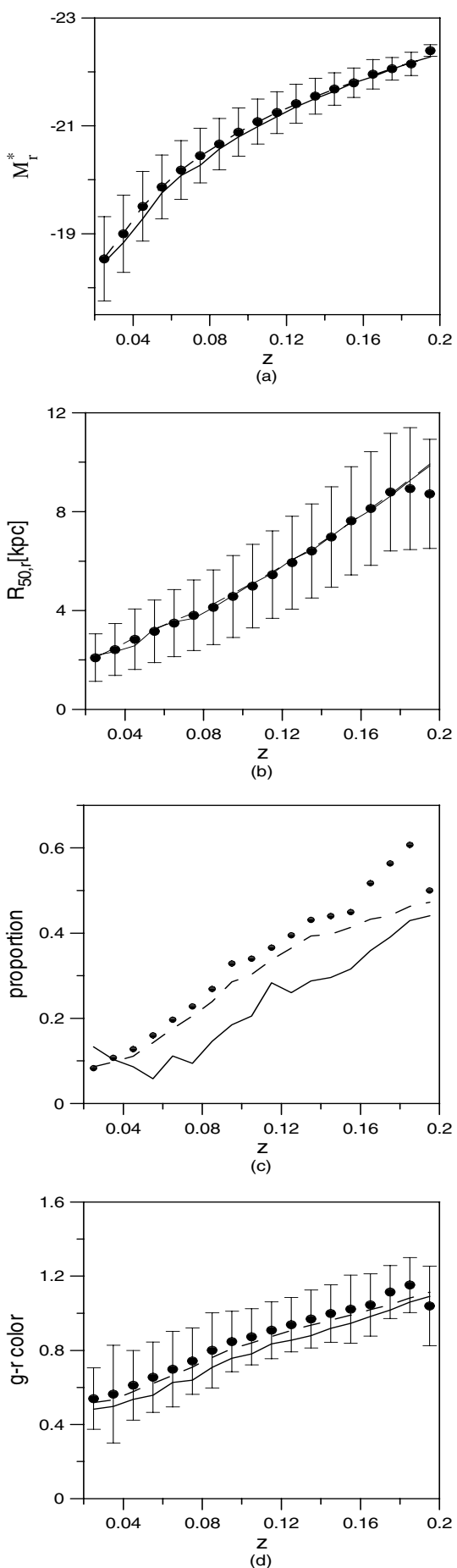

Fig. 8. Galaxy statistical properties as a function of redshift $z$ for member galaxies of groups identified at the linking length $b=0.2$ (dot), the main galaxy sample (dashed line) and isolated galaxies (solid line). Error bars represent standard deviation in each redshift bin for member galaxies of groups (a) mean luminosity, (b) mean size, (c) early-type proportion, (d) mean $g-r$ color.

show that more luminous galaxies exist preferentially in groups or clusters. Our method is more straightforward.

In Fig. 8b, we see that sizes of galaxies are nearly independent of environment. It is well-known that the galaxy sizes are correlated with luminosity (Kormendy 1977; Shen et al. 2003). Due to a weak dependence of galaxy luminosity on environment, we can explain this result.
It is widely accepted that galaxy morphologies seem to correlate (significantly) with environment: galaxies in dense environments (i.e. clusters or groups) have predominantly early type morphologies (e.g. Oemler 1974; Dressler 1980; Whitmore et al. 1993; Deng et al. 2007), while galaxies in the lowest density regions (isolated galaxies) have a lower proportion of early-type galaxies (e.g. Deng et al. 2006b). This suggests that in dense environments there is a transformation from late to early type. Many physical mechanisms, such as galaxy harassment (Moore et al. 1996), rampressure stripping (Gunn \& Gott 1972) and galaxy-galaxy merging (Toomre \& Toomre 1972) can explain such a process. In Fig. 8c, we notice the strong correlation between galaxy morphologies and environment. The difference of early-type proportion between isolated galaxies and the main galaxy sample is very large, while the difference between member galaxies of groups and the main galaxy sample is smaller. According to the morphology-density relation found by Goto et al. (2003): fractions of early-type galaxies increase with increasing local galaxy density, we infer that it may be due to quite low local density around isolated galaxies, while our groups are not dense enough to show a strong dependence of galaxy properties on enviroment.

Brown et al. (2000) and Zehavi et al. (2002) showed that clustering of galaxies strongly depends on color. Blanton et al. (2003b) found that local density is a strong function of all colors. However, there have been some different conclusions (e.g. Bernardi et al. 2003; Balogh et al. 2004a; Hogg et al. 2004). For example, Hogg et al. (2004) showed that red galaxy colors are independent of environment. Balogh et al. (2004a) found that at fixed luminosity the mean color of blue galaxies or red galaxies is nearly independent of environment, but at fixed luminosity the fraction of galaxies in the red distribution is a strong function of local density, increasing from $\sim 10-30 \%$ of the population in the lowest density environments, to $\sim 70 \%$ at the highest densities. They infered that most star-forming galaxies today evolve at a rate that is determined primarily by their intrinsic properties, and independent of their environment, and that the transformation from late to early type must be either sufficiently rapid, or sufficiently rare, to keep the overall color distribution unchanged. In Fig. 8d, we notice that on the average the $g-r$ color of member galaxies of groups is redder than that of isolated galaxies, but approximates that of the main galaxy sample. On the one hand this confirms the close correlation between environment and color, on the other hand it shows that in a dense environment the mean color of galaxies is only a weak function of local density.

\section{Summary}

Using the three-dimensional friends-of-friends (FOF) algorithm, we extracted 11163 galaxy groups with 4 or more members from the main galaxy sample of the SDSS Data Release 5, in which the richest group contains 113 galaxies. The linking length is defined as $R_{0}=0.2 \times \bar{n}^{-1 / 3}=1.97 \mathrm{Mpc}$, where $\bar{n}$ is the mean galaxy density. In order to study the correlations between galaxy properties and environment, we compare basic properties of member galaxies of groups with those of the main galaxy sample and isolated galaxies in each redshift bin, and find that luminosities and sizes of galaxies are nearly independent of environment, but morphologies and colors are correlated with environmentespecially galaxy morphologies.

Acknowledgements. We thank the referee, Professor Michael Strauss for many useful comments and suggestions.

Our study is supported by the Program for Innovative Research Team of Nanchang University. 
Funding for the creation and distribution of the SDSS Archive has been provided by the Alfred P. Sloan Foundation, the Participating Institutions, the National Aeronautics and Space Administration, the National Science Foundation, the U.S. Department of Energy, the Japanese Monbukagakusho, and the Max Planck Society. The SDSS Web site is http://www.sdss.org/

The SDSS is managed by the Astrophysical Research Consortium (ARC) for the Participating Institutions. The Participating Institutions are The University of Chicago, Fermilab, the Institute for Advanced Study, the Japan Participation Group, The Johns Hopkins University, Los Alamos National Laboratory, the Max-Planck-Institute for Astronomy (MPIA), the Max-Planck-Institute for Astrophysics (MPA), New Mexico State University, University of Pittsburgh, Princeton University, the United States Naval Observatory, and the University of Washington.

\section{References}

Abazajian, K., Adelman-McCarthy, J. K., Agüeros, M. A., et al. 2004, AJ, 128, 502

Abraham, R. G., van den Bergh, S., \& Nair, P. A. 2003, ApJ, 588, 218

Adams, M., Jensen, E., \& Stocke, J. 1980, AJ, 85, 1010

Adelman-McCarthy, J. K., Agüeros, M. A., Allam, S. S., et al. 2007, ApJS, submitted

Allam, S. S., Tucker, D. L., Lee, B. C., \& Smith, A. 2005, AJ, 129, 2062

Baldry, I. K., Glazebrook, K., Brinkmann, J., et al. 2004, ApJ, 600, 681

Balogh, M., Morris, S. L., Yee, H. K. C., et al. 1997, ApJ, 488, L75

Balogh, M., Morris, S. L., Yee, H. K. C., et al. 1999, ApJ, 527, 54

Balogh, M., Baldry, I. K., Nichol, R., et al. 2004a, ApJ, 615, L101

Balogh, M., Eke, V., Miller, C., et al. 2004b, MNRAS, 348, 1355

Benoist, C., Cappi, A., da Costa, L. N., et al. 1998, ApJ, 514, 563

Berlind, A. A., Frieman, J., Weinberg, D. H., et al. 2006, ApJS, 167, 1

Bernardi, M., Sheth, R. K., Annis, J., et al. 2003, AJ, 125, 1882

Blanton, M. R., Hogg, D. W., Bahcall, N. A., et al. 2003a, APJ, 594, 186

Blanton, M. R., Brinkmann, J., Csabai, I., et al. 2003b, AJ, 125, 2348

Bower, R. G., Lucey, J. R., \& Ellis, R. S. 1992, MNRAS, 254, 601

Brown, M. J. I., Webster, R. L., \& Boyle, B. J. 2000, MNRAS, 317, 782

Carlberg, R. G., Yee, H. K. C., Morris, S. L., et al. 2001, ApJ, 552, 427

Davis, M., Efstathiou, G., Frenk, C. S., \& White, S. D. M. 1985, ApJ, 292, 371

Davis, M., Meiksin, A., Strauss, M. A., et al. 1988, ApJ, 333, L9

Deng, X. F., Chen, Y. Q., Zhang, Q., et al. 2006a, ChJAA, 6, 35

Deng, X. F., Ma, X. S., Luo, C. H., et al. 2006b, PASA, 23, 76

Deng, X. F., He, J. Z., Jiang P., et al. 2007, IJMPD, 16, 885

de Vaucouleurs, G. 1961, ApJS, 5, 233

Diaferio, A., Ramella, M., Geller, M., et al. 1993, AJ, 105, 2035

Domínguez, M., Zandivarez, A., Martínez, H. J., et al. 2002, MNRAS, 335, 825

Dressler, A. 1980, ApJ, 236, 351

Dressler, A., Oemler, A. J., Couch, W. J., et al. 1997, ApJ, 490, 577

Einasto, J., Klypin, A. A., Saar, E., et al. 1984, MNRAS, 206, 529

Eke, V. R., Baugh, C. M., Cole, S., et al. 2004, MNRAS, 348, 866

Fasano, G., Poggianti, B. M., Couch, W. J., et al. 2000, ApJ, 542, 673

Fukugita, M., Ichikawa, T., Gunn, J. E., et al. 1996, AJ, 111, 1748

Geller, M. J., \& Huchra, J. P. 1982, ApJS, 52, 61

Gerke, B. F., Newman, J. A., Davis, M., et al. 2005, ApJ, 625, 5

Girardi, M., \& Giuricin, G. 2000, ApJ, 540, 45

Giuricin, G., Marinoni, C., Ceriani, L., \& Pisani, A. 2000, ApJ, 543,178

Giuricin, G., Samurović, S., Girardi, M., et al. 2001, ApJ, 554, 857

Gómez, P. L., Nichol, R. C., Miller, C. J., et al. 2003, ApJ, 584, 210

Goto, T., Yamauchi, C., Fujita, Y., et al. 2003, MNRAS, 346, 601

Gott, J. R. I., \& Turner, E. L. 1977, ApJ, 213, 309

Gott, J. R., Juric, M., Schlegel, D., et al. 2005, APJ, 624, 463

Gunn, J. E., \& Gott, J. R. I. 1972, ApJ, 176, 1

Gunn, J. E., Carr, M. A., Rockosi, C. M., et al. 1998, AJ, 116, 3040

Guzzo, L., Strauss, M. A., Fisher, K. B., et al. 1997, ApJ, 489, 37
Hamilton, A. J. S. 1988, ApJ, 331, L59

Hashimoto, Z., \& Oemler, A. 1999, ApJ, 510, 609

Hashimoto, Z., Oemler, A., Lin, H., et al. 1998, ApJ, 499, 589

Haynes, M. P., \& Giovanelli, R. 1980, ApJ, 240, L87

Haynes, M. P., Giovanelli, R., \& Chincarini, G. L. 1984, ARA\&A, 22, 445

Helsdon, S. F., \& Ponman, T. J. 2003, MNRAS, 339, L29

Hickson, P. 1982, ApJ, 255, 382

Hogg, D. W., Finkbeiner, D. P., Schlegel, D. J., et al. 2001, AJ, 122, 2129

Hogg, D. W., Blanton, M. R., Brinchmann, J., et al. 2004, ApJ, 601, L29

Hoyle, F., Rojas, R. R., Vogeley, M. S., \& Brinkmann, J. 2005, ApJ, 620, 618

Huchra, J. P., \& Geller, M. J. 1982, ApJ, 257, 423

Jackson, J. C. 1975, MNRAS, 173, 41p

Jenkins, A., Frenk, C. S., White, S. D. M., et al. 2001, MNRAS, 321, 372

Karachentseva, V. E. 1973, Soobshcheniya Spetsial'noj Astrofizicheskoj Observatorii, 8,3

Kelm, B., Focardi, P., \& Sorrentino, G. 2005, A\&A, 442, 117

Koopmann, R. A., \& Kenney, J. D. P. 1998, ApJ, 497, L75

Kormendy, J. 1977, APJ, 217, 406

Lee, B. C., Allam, S. S., Tucker, D. L., et al. 2004, AJ, 127, 1811

Lewis, I., Balogh, M., De Propris, R., et al. 2002, MNRAS, 334, 673

Loveday, J., Maddox, S. J., Efstathiou, G., et al. 1995, ApJ, 442, 457

Martínez, H. J., Zandivarez, A., Merchán, M. E., \& Domínguez, M. 2002, MNRAS, 337, 1441

Merchán, M. E., \& Zandivarez, A. 2002, MNRAS, 335, 216

Merchán, M. E., \& Zandivarez, A. 2005, ApJ, 630, 759

Merchán, M. E., Maia, M. A. G., \& Lambas, D. G. 2000, ApJ, 545, 26

Miller, C. J., Nichol, R. C., Reichart, D., et al. 2005, AJ, 130, 968

Moore, B., Katz, N., Lake, G., et al. 1996, Nature, 379, 613

Nakamura, O., Fukugita, M., Yasuda, N., et al. 2003, AJ, 125, 1682

Norberg, P., Baugh, C. M., Hawkins, E., et al. 2001, MNRAS, 328, 64

Norberg, P., Baugh, C. M., Hawkins, E., et al. 2002, MNRAS, 332, 827

Oemler, A. 1974, ApJ, 194, 1

Padilla, N. D., Baugh, C. M., Eke, V. R., et al. 2004, MNRAS, 352, 211

Park, C., \& Choi, Y. Y. 2005, ApJ, 635, L29

Park, C., Vogeley, M. S., Geller, M. J., et al. 1994, ApJ, 431, 569

Park, C., Choi, Y. Y., Vogeley, M. S., et al. 2005, ApJ, 633,11

Pier, J. R., Munn, J. A., Hindsley, R. B., et al. 2003, AJ, 125, 1559

Poggianti, B. M., Smail, I., Dressler, A., et al. 1999, ApJ, 518, 576

Postman, M., \& Geller, M. J. 1984, ApJ, 281, 95

Praton, E. A., Melott, A. L., \& Mckee, M. Q. 1997, ApJ, 479, L15

Ragone, C., Merchán, M. E., Muriel, H., \& Zandivarez, A. 2004, MNRAS, 350, 938

Ramella, M., Geller, M. J., Pisani, A., \& da Costa, L. N. 2002, AJ, 123, 2976

Rojas, R. R., Vogeley, M. S., Hoyle, F., \& Brinkmann, J. 2004, ApJ, 617, 50

Rojas, R. R., Vogeley, M. S., Hoyle, F., \& Brinkmann, J. 2005, ApJ, 624, 571

Rood, H. J., \& Dickel, J. R. 1978, ApJ, 224, 724

Shen, S. Y., Mo, H. J., White, S. D. M., et al. 2003, MNRAS, 343, 978

Shimasaku, K., Fukugita, M., Doi, M., et al. 2001, AJ, 122, 1238

Smith, J. A., Tucker, D. L., Kent, S. M., et al. 2002, AJ, 123, 2121

Stoughton, C., Lupton, R. H., Bernardi, M., et al. 2002, AJ, 123, 485

Strateva, I., Ivezic, Z., Knapp, G. R., et al. 2001, AJ, 122, 1861

Strauss, M. A., Weinberg, D. H., Lupton, R. H., et al. 2002, AJ, 124, 1810

Tanaka, M., Goto, T., Okamura, S., et al. 2004, AJ, 128, 2677

Toomre, A., \& Toomre, J. 1972, ApJ, 178, 623

Tran, K. H., Simard, L., Zabludoff, A. I., et al. 2001, ApJ, 549, 172

Treu, T., Ellis, R. S., Kneib, J., et al. 2003, ApJ, 591, 53

Tucker, D. L., Oemler, A. Jr., Hashimoto, Y., et al. 2000, ApJS, 130, 237

Turner, E. L., \& Sargent, W. L. W. 1974, ApJ, 194, 587

Whitmore, B. C., Gilmore, D. M., \& Jones, C. 1993, ApJ, 407, 489

Yamauchi, C., \& Goto, T. 2005, MNRAS, 359, 1557

York, D. G., Adelman, J., Anderson, J. E. Jr., et al. 2000, AJ, 120, 1579

Zandivarez, A., Merchán, M. E., \& Padilla, N. D. 2003, MNRAS, 344, 247

Zehavi, I., Blanton, M. R., Frieman, J. A., et al. 2002, ApJ, 571, 172 